\title{
El escudo de armas de Pedro Virgili en el Archivo de la Universidad de Cádiz
}

\author{
Francisco Glicerio Conde Mora \\ Doctor en Historia, Profesor del CUE Salus Infirmorum de Cádiz (Adscrito UCA)
}

RESUMEN: En el presente artículo analizamos el escudo de armas de Pedro Virgili, fundador del Real Colegio de Cirugía de la Armada de Cádiz que encontramos en el Archivo de la Universidad de Cádiz, armas concedidas a Virgili por el rey Fernando VI. Además de en el Archivo de la UCA, su heráldica se encuentra también en su localidad natal Vilallonga del Camp, en la provincia de Tarragona y en el Archivo de la Corona de Aragón.

Palabras claves: Virgili, Heráldica, Nobleza, Cirujano.

\section{The Pedro Virgili's coat of arms in the Archive of the University of Cádiz.}

ABSTRACT: In this article we analyze the coat of arms of Pedro Virgili, founder of the Real Colegio de Cirugía de la Armada de Cádiz that we find in the Archivo de la Universidad de Cádiz, coat of arms granted to Virgili by King Ferdinand VI. In addition to the Archivo UCA, its heraldry is also found in its hometown of Vilallonga del Camp, in the province of Tarragona and in the Archivo de la Corona de Aragon.

Keywords: Virgili, Heraldry, Nobility, Surgeon.

\section{BREVE RESEÑA BIOGRÁFICA DE D. PEDRo VIRGILI}

Pedro Virgili nació a finales del reinado de Carlos II El Hechizado, en 1699, en Vilallonga del Camp, en la provincia de Tarragona ${ }^{1}$. En 1748, junto a Jean Lacombe (Juan

\footnotetext{
${ }^{1}$ En relación a sus orígenes familiares consúltese Trenchs OdEnA, J., Documents de la familia d'en Pere Virgili a Vilallonga del Camp. Butlletí Arqueològic. Reial Societat Arqueològica Tarraconense, (133-140). 1967, pp. 237-250. Sobre Pedro Virgili pueden consultarse también los siguientes estudios: FERRER, D., 1963 Biografía de Pedro Virgili. Barcelona. Emporium; y Orozco Acuaviva, A., Pedro Virgili y el Hospital de Cádiz: en el bicentenario de la muerte del fundador del Real Colegio de Cirugía de Cádiz. Medicina \& Historia: Revista de estudios históricos de las ciencias médicas. España: 1967, n. ${ }^{\circ}$ 63, pp. 7-26 y del mismo autor: Pedro Virgili, cirujano de la Armada. Revista de historia naval. Madrid: Servicio de Publicaciones de la Armada, 2000, n. ${ }^{\circ} 69$, pp. 85-96.
}

DOI del artículo:

https://doi.org/10.25267/Cuad investig fondos arch UCA.2020.i2.04 
Lacomba), fundó el Real Colegio de Cirugía de la Armada de Cádiz ${ }^{2}$. En este centro de formación de la Armada se formaron prestigiosas figuras de la Sanidad Española de los siglos XVIII y XIX ${ }^{3}$. Desempeñó los cargos de Cirujano Mayor de la Armada y Médico de la Real Cámara. Fue también el creador del Real Colegio de Cirugía de Barcelona en 1760, ciudad donde tuvo lugar su óbito en $1776^{4}$. Remitimos al excelente estudio publicado por el Dr. José Manuel Blanco-Villero en el Boletín de la Real Academia de Hispanoamérica sobre nuestro personaje, titular del escudo de armas que se conserva en el Archivo de la Universidad y a continuación analizaremos ${ }^{5}$.

Figura 1. Retrato de Pedro Virgili

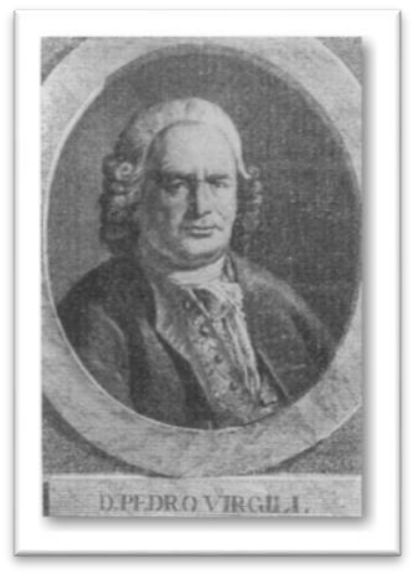

Fuente: Biblioteca Nacional (BNE)

${ }^{2}$ Existen excelentes trabajos publicados sobre el Real Colegio de Cirugía. Destacamos: FERRER, D., y ROMERO Abreu, J., 1961. Historia del Real Colegio de Cirugía de la Armada de Cádiz: ed. en honor de Pedro Virgili... Colegio Oficial de Médicos y LóPez Rodríguez, A., 1969. El Real Colegio de Cirugía de Cádiz y su época: discurso leído en la solemne apertura del curso académico de 1969-70 en la Universidad de Sevilla. Universidad de Sevilla. Destacar igualmente los trabajos de Manuel Bustos: Bustos RodríGuEZ, M., 1983. Los Cirujanos del Real Colegio de Cádiz en la encrucijada de la Ilustración, (1748-1796). Editorial: Universidad de Cádiz. Servicio de Publicaciones y más recientemente Bustos Rodríguez, M., Capítulo 7. El Hospital Real y el Real Colegio de Cirugía en la Edad Moderna. En: DARÍo Bernal CASASOlA (ed. lit.), JosÉ Manuel VArgas Girón (ed. lit.), Macarena lara Medina (ed. lit.) 7 metros de la historia de Cádiz...: arqueología en el Olivillo y en el Colegio Mayor Universitario. Cádiz: Universidad de Cádiz, 2019, pp. 329350. ISBN 978-84-9828-738-7. Para entender la importancia de la labor de los Reales Colegios de Cirugía en el siglo XVIII remitimos a Orozco ACuAviva, A., 2001. Los Reales Colegios de Cirugía en España. En: Rodríguez Pérez, M. E. y (coord.) MArtínez BArbosa, X. (eds.), Medicina NovoHispana siglo XVIII. México: Universidad Autónoma de México, Historia General de la Medicina de México, pp. 385-392. ISBN 968-36-9801-8.

3 Anexo al Real Colegio de Cirugía se encontraba el Hospital Real (fundado en el siglo XVII). MONTERO Carmona, J. Ma, 2018. El Hospital Real de Cádiz. 1667-1984. Ateneo: revista cultural del Ateneo de Cádiz, (18), 107-115. Se espera la tesis doctoral de D. José $\mathbf{M}^{\mathrm{a}}$ Montero Carmona que aportará numerosa documentación inédita sobre este nosocomio castrense.

${ }^{4}$ Sobre el Real Colegio de Cirugía de Barcelona, destinado a formar facultativos para el Ejército (al igual que el de Cádiz lo fue para la Armada) véase Usandizaga Soraluce, M., 1964. Historia del Real Colegio de Cirugía de Barcelona (1760-1843).

${ }^{5}$ Blanco-Villero, J. M., 2018. Pedro Virgili y el Real Colegio de Cirugía de Cádiz. Revista de la Real Academia Hispano Americana, (8), 13. 


\section{DESCRIPCIÓN HERÁLDICA DE SUS ARMAS}

En el Archivo de la Universidad de Cádiz encontramos su escudo de armas en el libro «Títulos de Nobleza concedidos a Don Pedro Virgili por S.M Católica Don Fernando VI, 1754-1756». Fue el rey Fernando VI (1746-1759), hijo y sucesor en 1746 de Felipe V, quién honró a Pedro Virgili por sus servicios nombrándole Médico de Cámara y distinguiéndole con una patente de nobleza para sí y sus descendientes, ciñéndole la espada de caballero $\mathrm{S}$. M. por su propia mano y concediendo a su escudo y cuarteles ${ }^{6}$.

La descripción de las armas de Pedro Virgili es la siguiente «de azur con contrabanda de plata cargada con tres leones rampantes de su color y acompañada de ocho flores de lis de oro».

Figura 2. Títulos de Nobleza concedidos a Don Pedro Virgili por S.M Católica Don Fernando VI, 17541756

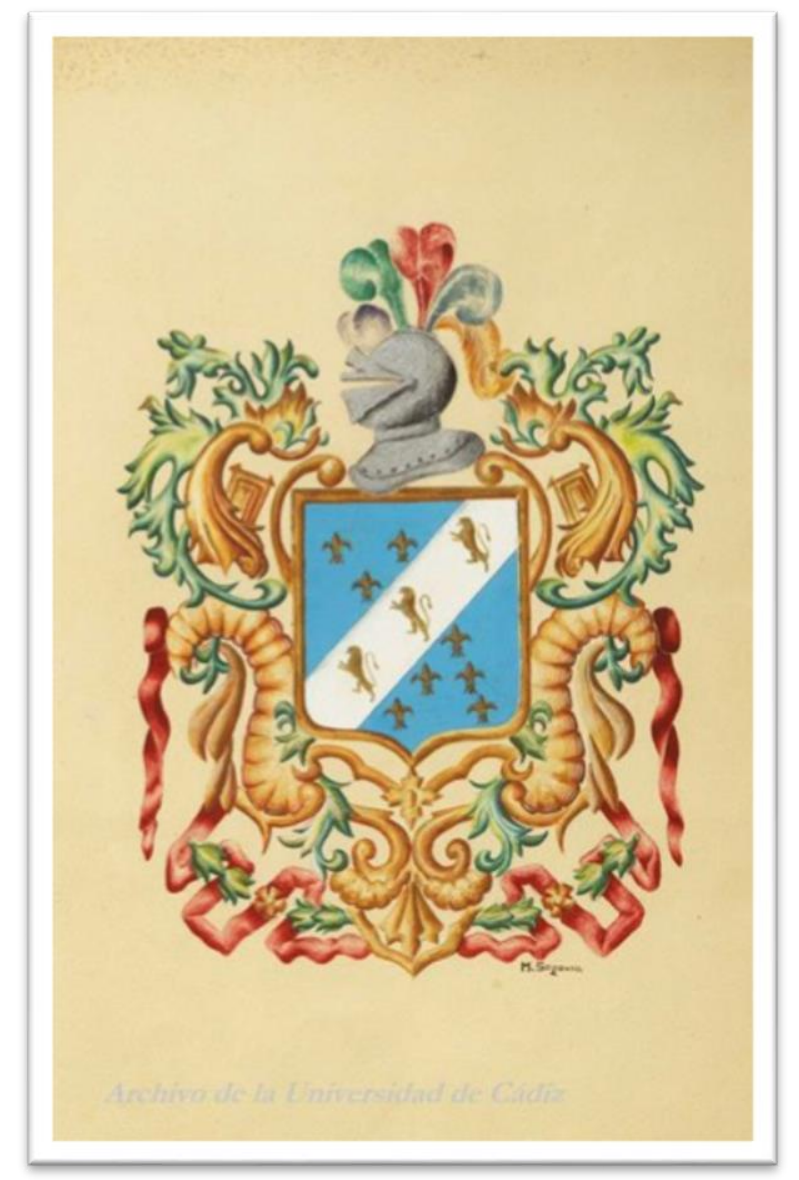

Fuente: Archivo de la Universidad de Cádiz (AUCA). L-61 RC

${ }^{6}$ Archivo de la Universidad de Cádiz (AUCA). Libro «Títulos de Nobleza concedidos a Don Pedro Virgili por S. M. Católica Don Fernando VI, 1754-1756». Sobre la importancia para la investigación de los fondos que conserva el Archivo de la UCA remitimos al trabajo publicado en el número precedente de esta publicación por el Dr. Alberto Ramos: Ramos SantanA, A., 2019. Los fondos históricos del Archivo de la Universidad de Cádiz: bastante más que historia de la universidad. Cuadernos de Investigación de Fondos del Archivo UCA [en línea]. Cádiz: Editorial UCA, n. ${ }^{\circ}$ 1, p. 47. ISSN 2659-4951. Disponible en: https://revistas.uca.es/index.php/cifa/issue/view/360. 
En el Archivo de la Corona de Aragón localizamos también documentación similar a estas armas de Pedro Virgili que encontramos en el Archivo de la Universidad de Cádiz (AUCA). No es un registro de expedición, sino un copiador de los privilegios reales de ciudadano honrado, caballero y nobleza presentados ante la Audiencia para su reconocimiento público en Cataluña. Contiene varios privilegios expedidos y presentados ante la Audiencia durante el reinado de Carlos III, entre los años 1759 y 1774.

En comparación con el documento conservado en el Archivo de la Universidad de Cádiz, los conservados en el de la Corona de Aragón se encuentran en mal estado. Son varios los folios muy oxidados por causa de las tintas ferrogálicas. Su Título original en el folio 1r: «Registro del Privilegiorum primo Regia Audiencia del reynado del señor Don Carlos tercero». En el folio 87r encontramos el de Pedro Virgili ${ }^{7}$.

Figura 3. Escudo de armas de Pedro Virgili

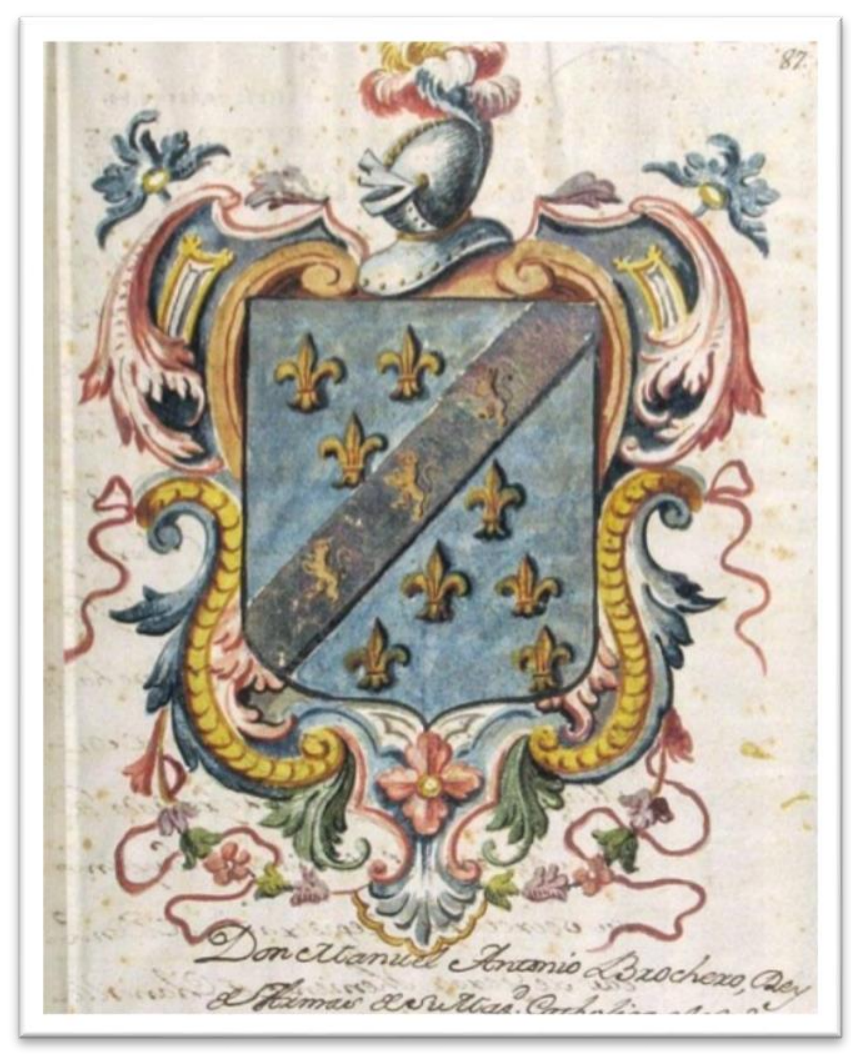

Fuente: Archivo de la Corona de Aragón, REAL AUDIENCIA, Registros, 990, f.o 87r

También encontramos las armas de Pedro Virgili en un Monumento a él dedicado en su municipio natal Vilallonga del Camp, en la provincia de Tarragona. Sin embargo, no debemos confundir estas armas con las del Real Colegio de Cirugía que Virgili fundó en Cádiz en 1748.

Por una carta fechada en 1749 de Pedro Virgili a Alonso Pérez Delgado que se conserva en el Archivo General de Simancas (AGS) conocemos uno de los primeros diseños de las armas para la institución fundada por Virgili tan sólo un año antes.

${ }^{7}$ Archivo de la Corona de Aragón, Real Audiencia, Registros, 990, f. ${ }^{\circ}$ 87r.

DOI del artículo:

https://doi.org/10.25267/Cuad investig fondos arch UCA.2020.i2.04 
Como puede verse en la figura 4, se trata de un diseño de forma cilíndrica con corona que incluye los atributos de la cirugía: En primer lugar Prudencia, en segundo la Farmacia, en tercer lugar el Hierro y por último el Fuego ${ }^{8}$.

Figura 4. Escudo del Real Colegio de Cirugía de la Armada de Cádiz

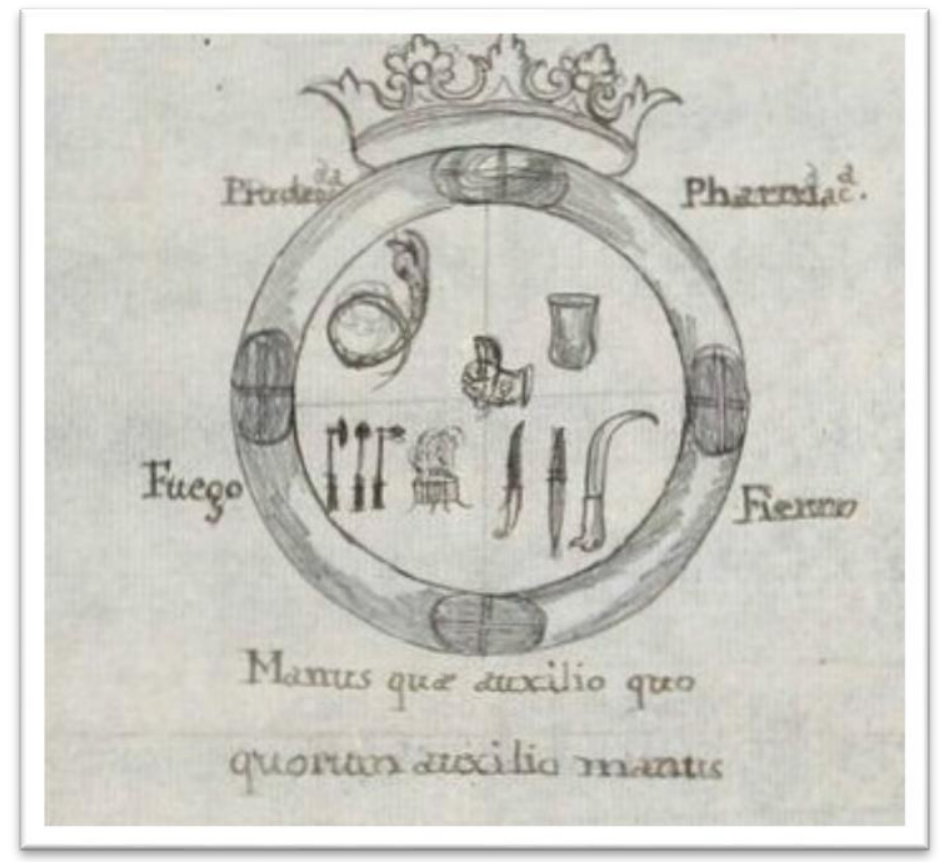

Fuente: Archivo General de Simancas (AGS) Secretaría de Marina, 00219

En la figura 6 puede observarse el escudo del Colegio de Cirugía de Cádiz, la mano con un ojo en la palma y la leyenda «MANU QUA AUXILIO QUO». La confusión de este escudo con el de Virgili procede de la obra de Anastasio Chinchilla titulada Anales históricos de la Medicina en general, y biográfico-bibliográfico de la española en particular, en la que identifica como armas de Virgili las propias del Real Colegio

... Le eligió por cirujano de su real cámara, y le distinguió con el titulo de nobleza para si y sus descendientes: S. M. le cinó por su [...] El escudo de sus armas es una mano abierta con un ojo en la palma, y un lazo en la muñeca con el lema siguiente: manu qua, auxilio quo, y una corona real encima del escudo ${ }^{9}$.

El célebre médico militar Mariano Gómez Ulla en su trabajo La Cirugía en la guerra publicado en 1942 hace referencia a las armas de D. Pedro Virgili, afirmando que se conservan en la Facultad de Medicina de Cádiz ${ }^{10}$.

\footnotetext{
${ }^{8}$ Archivo General de Simancas (AGS). Secretaría de Marina, 00219

${ }^{9}$ Chinchilla PIQUeRAs, A., 1846. Anales históricos de la Medicina en general, y biográfico-bibliográfico de la española en particular p. 420.

${ }^{10}$ Se trata del discurso leído el 29 de abril de 1942 para su recepción como Académico en la Real Academia de Medicina. Gómez Ulla, M., 1942. La Cirugía en la guerra. Real Academia Nacional de Medicina, p. 21. Aunque Gómez Ulla remite al documento conservado en el AUCA equivoca al igual que Chinchilla un siglo antes las armas de D. Pedro Virgili con las de la Real institución que fundó, el Real Colegio de Cirugía de la
} Armada. 


\section{Las armas de Pedro Virgili en el Archivo de la Universidad de Cádiz}

Figura 5. Portada del libro de Diego Ferrer Historia abreviada del Real Colegio Cirugía de la Armada de Cádiz, donde puede verse las armas del Real Colegio que Pedro Virgili fundó.

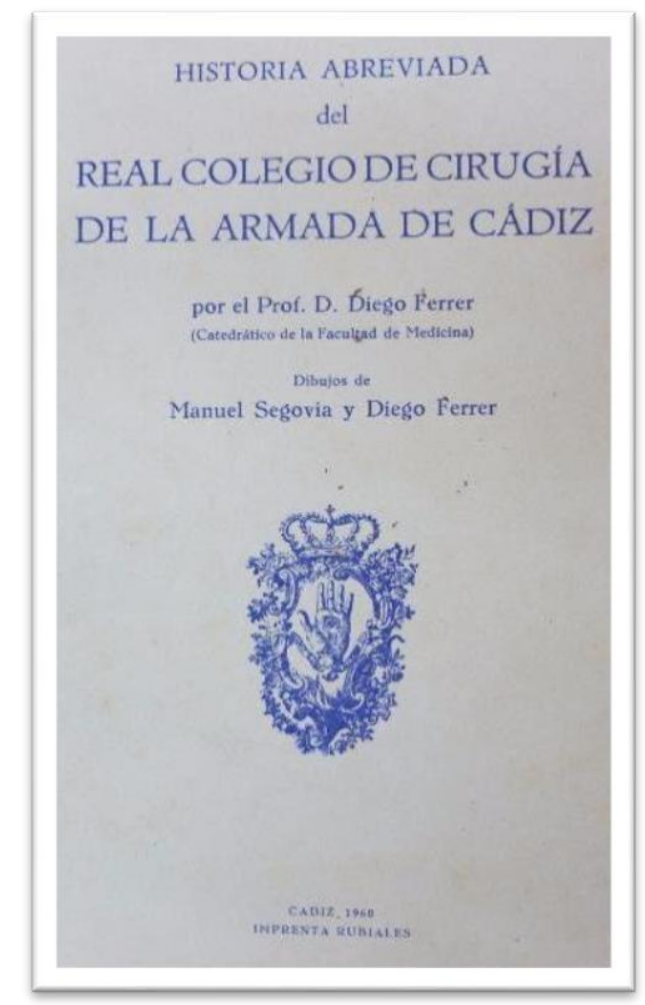

Figura 6. Escudo de armas del Real Colegio

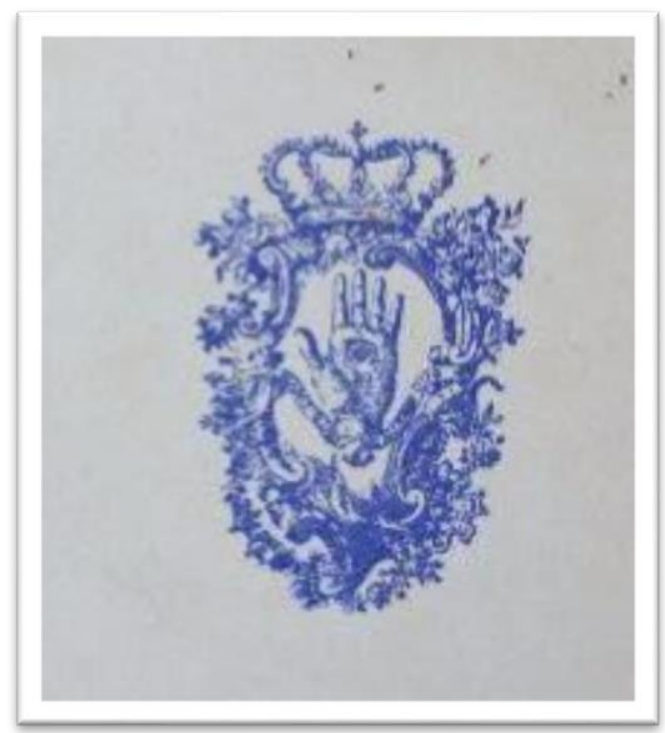

\section{CONClusiones}

Las armas de Pedro Virgili en los fondos documentales del Archivo Histórico de la UCA son un valioso testimonio heráldico. Como hemos podido ver estas armas fueron el reconocimiento del rey Fernando VI (1746-1759) a la excelente labor de una vida dedicada a 
la Sanidad, siéndole concedido por ello las citadas armas, reproducidas en la figura 2. Recordemos, por último, que todavía a día de hoy podemos encontrar estas armas en la Facultad de Medicina (heredera del Real Colegio de Cirugía de la Armada), detrás del busto que preside su entrada.

Figura 7. Busto de Pedro Virgili

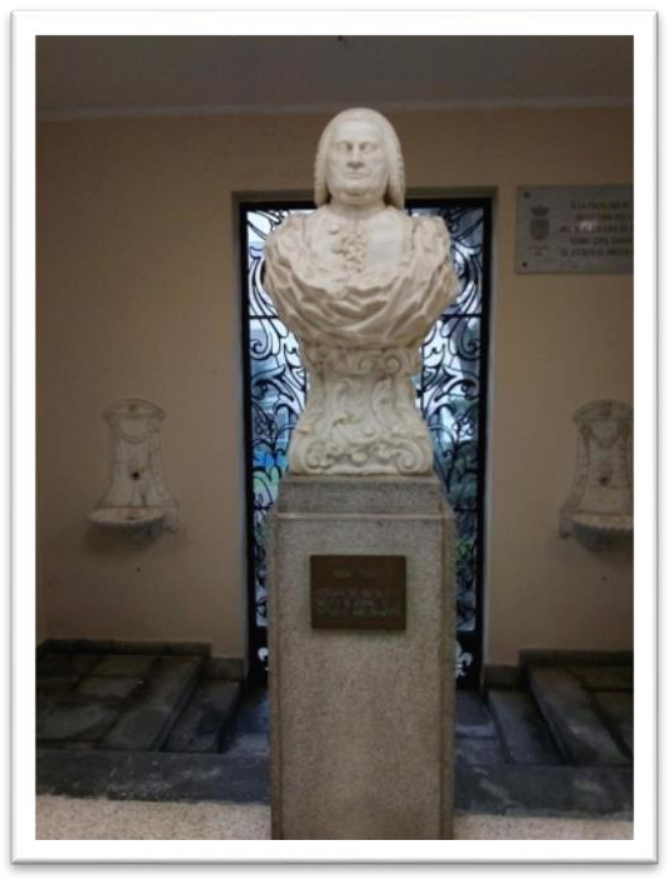

Fuente: Fotografía realizada por el autor
Figura 8. Fotografía de una puerta con el escudo de armas.

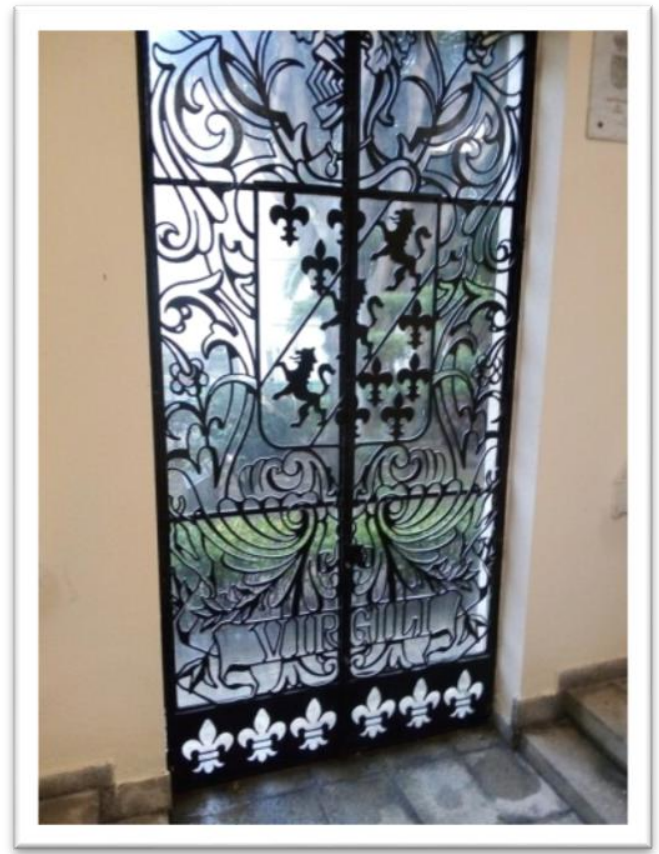

Fuente: Fotografía realizada por el autor

\section{FUENTES DOCUMENTALES}

Archivo de la Universidad de Cádiz (AUCA). Libro Títulos de Nobleza concedidos a Don Pedro Virgili por S.M Católica Don Fernando VI, 1754-1756. L-61 RC.

Archivo de la Corona de Aragón, Real Audiencia, Registros, 990, f. ${ }^{\text { 87r. }}$

Archivo General de Simancas (AGS). Secretaría de Marina, 00219. Cádiz, 3 de febrero de 1749.

\section{BIBLIOGRAFÍA}

APPOLIS, E., 1962. Un grand chirurgien espagnol ancién étudiant a Montpellier. Don Pedro Virgili (1699-1776), en Monspeliensis Hippocrates, 6.

BECH I BORRÀs, J., 1990. Sobre le privilegi de nosblesa de Castelà i" fuero de hijo dalgo" concedits per Ferran VI a Virgili i l'ús d'aquests a Barcelona. Gimbernat: revista catalana d'història de la medicina i de la ciència, 14, pp. 49-60.

Blanco-Villero, J. M., 2018. Pedro Virgili y el Real Colegio de Cirugía de Cádiz. Revista de la Real Academia Hispano Americana, (8), 13. 
Bustos Rodríguez, M., 1983. Los Cirujanos del Real Colegio de Cádiz en la encrucijada de la Ilustración, (1748-1796). Editorial: Universidad de Cádiz. Servicio de Publicaciones.

Bustos Rodríguez, M., Capítulo 7. El Hospital Real y el Real Colegio de Cirugía en la Edad Moderna. En: Darío Bernal Casasola (ed. lit.), José Manuel Vargas Girón (ed. lit.), MACARENA LARA MEDINA (ed. lit.) 7 metros de la historia de Cádiz...: arqueología en el Olivillo y en el Colegio Mayor Universitario. Cádiz: Universidad de Cádiz, 2019, pp. 329-350. ISBN 978-84-9828-738-7.

Comenge y Ferrer, L., 1893. Apuntes para la biografía de Pedro Virgili. Barcelona, Heinrich y Cía.

Chinchilla, A. 1846, Anales históricos de la Medicina en general, y biográficobibliográfico de la española en particular.

Ferrer, D., Y Romero Abreu, J., 1961. Historia del Real Colegio de Cirugía de la Armada de Cádiz, ed. en honor de Pedro Virgili... Cádiz: Colegio Oficial de Médicos.

FERrer, D., 1963. Biografía de Pedro Virgili. Barcelona. Emporium.

García Cubillana de la CruZ, J. M., 2018. La sanidad de la Armada en la Bahía de Cádiz en el siglo XVIII. Revista de la Real Academia Hispano Americana, (8), 12.

Gómez Ulla, M. 1942. La Cirugía en la guerra. Real Academia Nacional de Medicina.

López Rodríguez, A., 1969. El Real Colegio de Cirugía de Cádiz y su época: discurso leído en la solemne apertura del curso académico de 1969-70 en la Universidad de Sevilla. Universidad de Sevilla.

Montero Carmona, J. Mª 2018. El Hospital Real de Cádiz. 1667-1984. Ateneo: revista cultural del Ateneo de Cádiz, (18), 107-115.

Orozco Acuaviva, A., 1976. Pedro Virgili y el Hospital de Cádiz: en el bicentenario de la muerte del fundador del Real Colegio de Cirugía de Cádiz. Medicina \& Historia: Revista de estudios históricos de las ciencias médica. España: 1976, n. ${ }^{\circ}$ 63, pp. 7-26.

Orozco Acuaviva, A., 2000. Pedro Virgili, cirujano de la Armada. Revista de historia naval, n. ${ }^{\circ}$ 69, pp. 85-96.

Orozco Acuaviva, A. Los Reales Colegios de Cirugía en España. En: M. E. Rodríguez PÉreZ y X. (coord.) MARTíneZ BARbosa (eds.), Medicina NovoHispana siglo XVIII. México: Universidad Autónoma de México, Historia General de la Medicina de México, 2001, pp. 385-392. ISBN 968-36-9801-8.

Ramos Santana, A., 2019. Los fondos históricos del Archivo de la Universidad de Cádiz: bastante más que historia de la universidad. Cuadernos de Investigación de Fondos del Archivo UCA [en línea]. Cádiz: Editorial UCA, n. ${ }^{o}$ 1, p. 47. ISSN 2659-4951. Disponible en: https://revistas.uca.es/index.php/cifa/issue/view/360.

Trenchs Odena, J., 1977. Documents de la familia d'en Pere Virgili a Vilallonga del Camp. Butlletí Arqueològic. Reial Societat Arqueològica Tarraconense, (133-140). 1967, pp. 237-250.

Usandizaga Soraluce, M., 1964. Historia del Real Colegio de Cirugía de Barcelona (1760-1843). 